\section{Microtubule bundling}

SIR-We recently described the bundling of microtubules into parallel arrays by microtubule-associated proteins MAP2 and tau, as well as experiments that led to a model of how this process might occur (S. A. Lcwis et al. Nature 342, 498-505; 1989). Our model depicted the formation of crossbridges between closely spaced parallel microtubules via self-interactions between a short hydrophobic $\alpha$-helical region at the extreme carboxy terminus of MAP2 and tau. Our reasons for proposing the model were based on the following observations:(1) deletion of the hydrophobic $\alpha$-helical domain of MAP2 eliminated the ability of the molecule to bundle microtubles without affecting microtubule binding;(2) substitution of this hydrophobic $\alpha$-helical domain with the yeast GCN4 sequence (which is known to form dimers) restored the capacity to form microtubule bundles; and (3) a synthetic peptide corresponding to the hydrophobic $\alpha$-helical domain of MAP2 behaved in many respects as a dimer.

Because the crosslinking of microtubules into bundles seemed to depend on the presence of a zipper-like hydrophobic domain, we decided to assay the microtubule-bundling ability of molecules containing amino-acid substitutions that we predicted would destroy the $\alpha$-helical structure of the carboxy-terminal region. Contrary to our expectations, mutations in this domain (FSZ, Fig. 1) yielded phenotypes indistinguishable from that of the wild-type molecule: all were capable of bundling microtubules to the same extent. As this experiment cast doubt on our proposed mechanism for microtubule bundling, we made 12 constructs deleting various sequences from the region of MAP2 encoding the binding/bundling domain and assayed the expressed proteins for their ability to bind to and bundle microtubules in transient transfection assays.

Although all of the new constructs led to the synthesis of properly terminated proteins, the strategem used in the construction of some of the clones (FS-H and FS-C) described in our paper led to the expression of proteins that included 29 extraneous amino acids at the carboxy terminus (zig-zag line, Fig. 1). These were encoded by a stretch of the 3' untranslated region of the MAP2 messenger RNA. Although this extraneous sequence has no effect on the results obtained with FS-C (compare FS-C with FS66, Fig. 1), it exerts a profound effect on the results

Binding Bundling

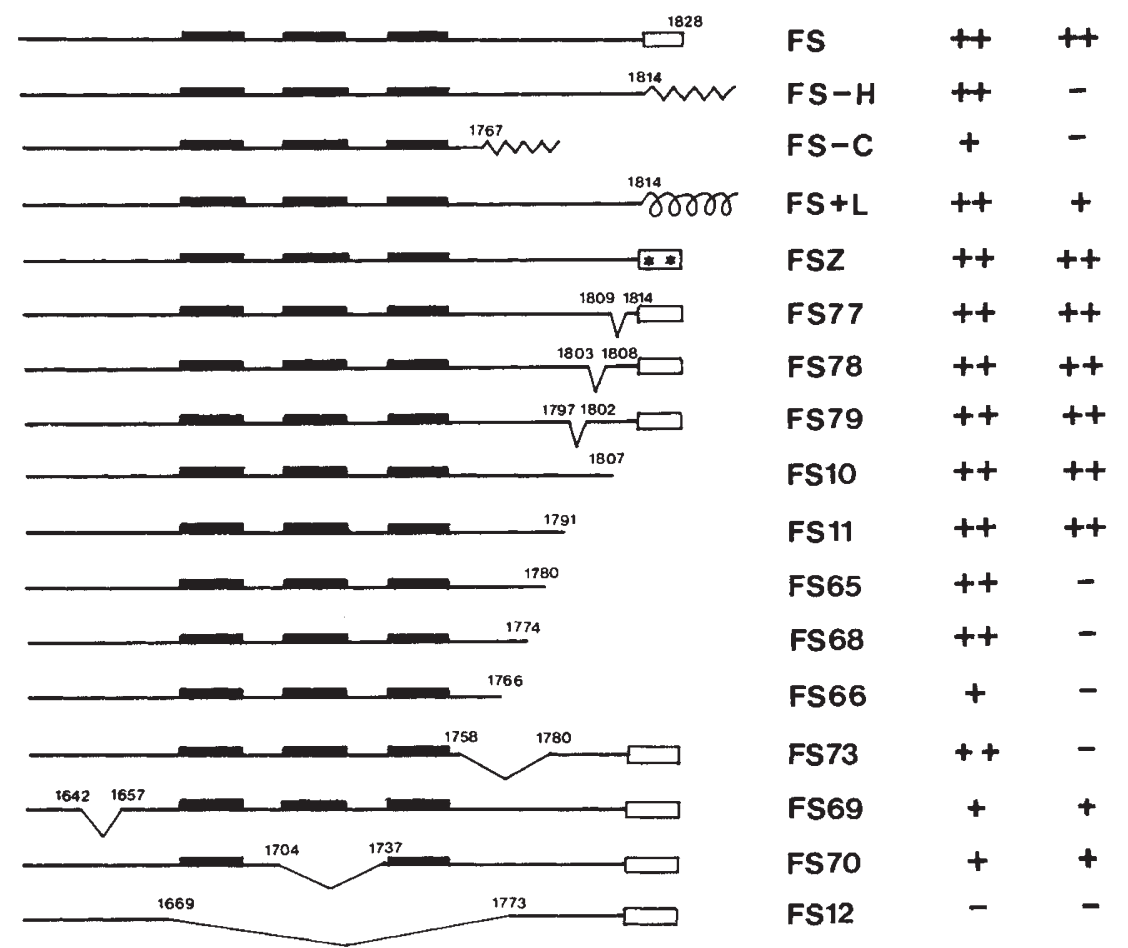

FIG. 1 Ability of MAP2 constructs to bind to and bundle microtubules. All constructs were derived from FS, a deleted version of MAP2 lacking amino-acids 228-1,621 that gives a phenotype upon transfection into cultured cells that is indistinguishable from intact MAP2. Only the region Cterminal to amino acid 1,621 is shown. Raised solid bars, three imperfect repeats forming part of the microtubule-binding domain. Open boxes, hydrophobic MAP2 carboxy-terminal $\alpha$-helix. Amino-acid residue numbers at the deletion end-points of each construct are shown. Zig-zag, extraneous extension of 29 amino acids translated from constructs FS-H and FS-C; $a$ helix that is part of the $\mathrm{FS}+\mathrm{L}$ construct denotes the leucine zipper domain derived from the yeast GCN4 protein. Asterisks in FSZ, amino-acid substitutions introduced into the hydrophobic carboxy terminus to disrupt the $\alpha$-helical structure. See our paper for further details.

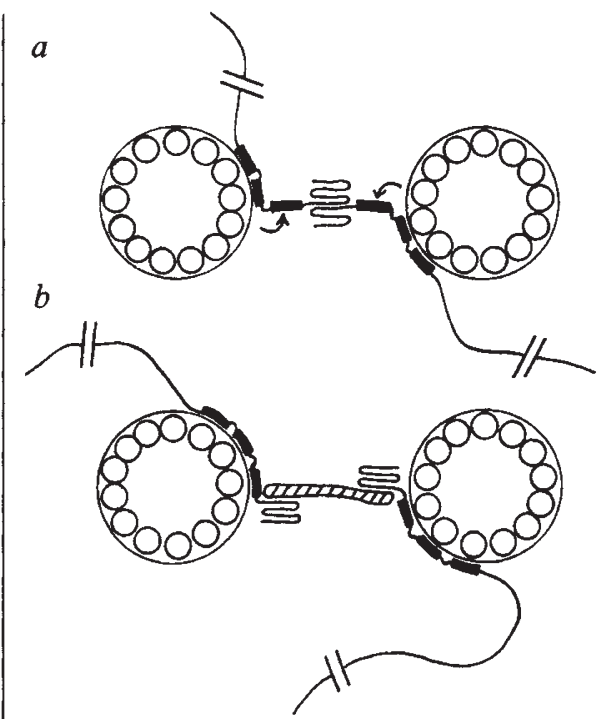

FIG. 2 Microtubule bundling by MAP2. Crossbridged microtubles with a $20 \mathrm{~nm}$ spacing are shown. Solid areas, imperfect repeats of the microtubule-binding domain; carboxy terminus, folded region. Hatched region in $b$ is a bridging molecule unrelated to MAP2.

obtained with FS-H and leads to a complete suppression of bundling (compare FS-H with FSZ, FS10, FS11, Fig. 1). Given these new data, it is clear that the carboxy-terminal helix of MAP2 is not directly responsible for microtubule bundling as we originally proposed. The observation that substitution of this hydrophobic $\alpha$-helix with the leucine zipper from GCN4 results in a construct whose expression causes microtubule bundling (FS $+\mathrm{L}$, Fig. 1) must therefore be reinterpreted: the appended leucine zipper is evidently less destructive to the folding of some critical region than is the 29-amino-acid extension present in FS-H. In addition, the tendency of a synthetic peptide corresponding to the C-terminal $\alpha$-helix to dimerize in vitro must be irrelevant to the microtubule-bundling mechanism.

Our new data indicate that amino acids $1,758-1,780$ of MAP2 are involved in microtubule bundling either directly or by influencing the secondary structure of a critical region. Two ways in which these amino acids could contribute to microtubule bundling are depicted in Fig. 2. In $a$, sequences dimerize to bridge microtubules, whereas in $b$ they interact with a bridging protein. In case $a$ it is necessary to postulate that the third repeat is detached from the microtubule, to give a bridge consistent with the length of crossbridges observed in electron micrographs of microtubule bundles in transfected cells. Such a mechanism could restrict bundling to regions of high microtubule density, as dissociation of a repeat would be expected to destabilize binding.

Sally A. LeWIS NICHOLAS COWAN

Department of Biochemistry,

New York University Medical Center,

New York, New York 10016, USA 